\title{
A Case of Myxoid Dermatofibrosarcoma Protuberans
}

\author{
Yeon Ju Hong, M.D., You Won Choi, M.D., Ki Bum Myung, M.D., Hae Young Choi, M.D. \\ Department of Dermatology, School of Medicine, Ewha Womans University, Seoul, Korea
}

\begin{abstract}
Dermatofibrosarcoma protuberans (DFSP) is a slowly growing dermal spindle cell tumor and its myxoid variant, a rare type of DFSP, is characterized by extensive myxoid degeneration. We present the case of a 69-year-old woman with a multinodular reddish plaque on her trunk. Histopathologically, the tumor was located in the dermis and consisted of uniform spindle-shaped cells, showing strongly positive reaction for CD34, and negative for both S-100 and desmin. In addition to the typical storiform pattern, prominent myxoid stromal changes were demonstrated. Herein, we report an interesting case of myxoid DFSP, rarely reported in the dermatology literature. (Ann Dermatol 23(3) 379 381, 2011)
\end{abstract}

\section{-Keywords-}

Dermatofibrosarcoma protuberans, Myxoid variant

\section{INTRODUCTION}

Dermatofibrosarcoma protuberans (DFSP) is a rare, locally aggressive dermal mesenchymal neoplasm. The characteristic histologic feature of DFSP is the proliferation of densely packed monomorphous spindle cells, arranged in a storiform pattern. To date, various histological patterns, including the myxoid, fibrosarcomatous and granular cell types, have been described in the English literature ${ }^{1}$, but myxoid variant of DFSP has not yet been reported in the

Received April 26, 2010, Revised July 21, 2010, Accepted for publication July 21, 2010

Corresponding author: Hae Young Choi, M.D., Department of Dermatology, Ewha Womans University Mokdong Hospital, 911-1 Mok-dong, Yangcheon-gu, Seoul 158-710, Korea. Tel: 82-2-26505159, Fax: 82-2-2652-6925, E-mail: hychoi@ewha.ac.kr

This is an Open Access article distributed under the terms of the Creative Commons Attribution Non-Commercial License (http:// creativecommons.org/licenses/by-nc/3.0) which permits unrestricted non-commercial use, distribution, and reproduction in any medium, provided the original work is properly cited.
Korean literature. Herein, we report a case of myxoid DFSP, rarely reported in the Korean dermatology literature.

\section{CASE REPORT}

A 69-year-old woman was presented with an enlarging nodular lesion on her trunk. The lesion had been growing over a period of 6 months and she denied any discomfort, except the occasional itching sensation. There was no history of trauma or any preexisting skin lesions in the affected area. Clinical examination revealed multiple erythematous to brownish nodules, with reddish plaque on her abdomen, and some part of the lesion had a gelatinous appearance (Fig. 1).

A skin biopsy was performed and the lesion showed a diffusely infiltrating tumor, involving the entire dermis (Fig. 2). In some parts of the lesion (Fig. 3A), the tumor showed the characteristic storiform pattern, but most of the tumor cells were randomly embedded in the palely eosinophilic stroma (Fig. 3B). The tumor consisted of relatively uniform spindle cells, with elongated nuclei (Fig $3 C)$, and the stroma was diffusely positive for the alcian-

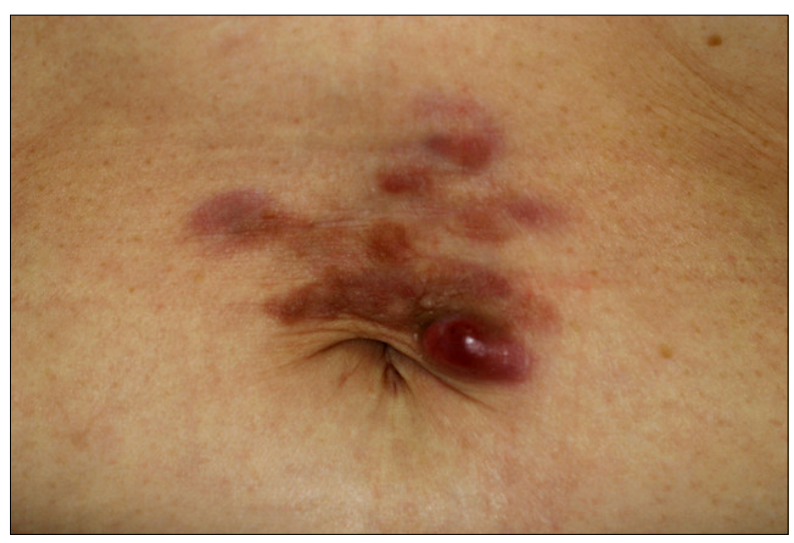

Fig. 1. Multinodular reddish plaques, with partial gelatinous appearance on the abdomen. 
blue stain, indicating the prominent myxoid degeneration. Immunohistochemically, the tumor cells were strongly positive for CD34 (Fig. 4). Other markers, including desmin, S-100, and epithelial membrane antigen (EMA), were all negative.

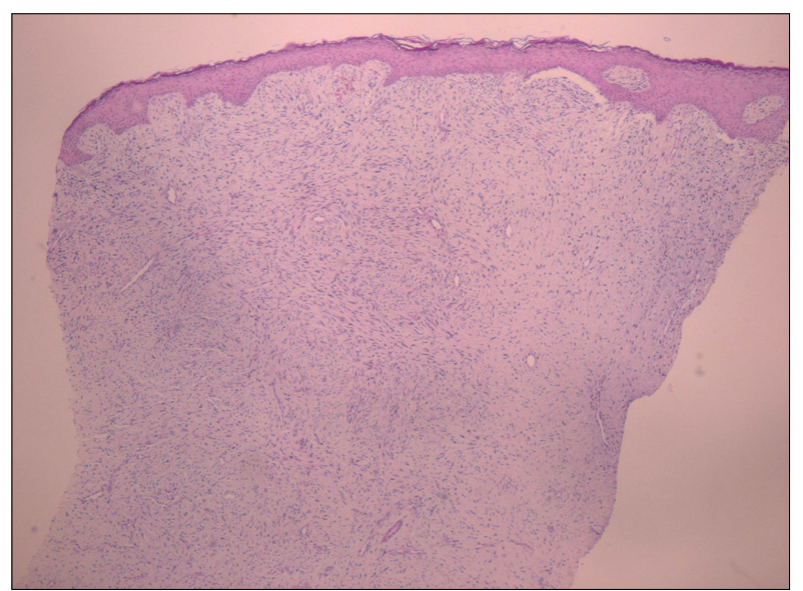

Fig. 2. The diffusely infiltrating tumor was located in the entire dermis $(\mathrm{H} \& \mathrm{E}, \times 40)$.
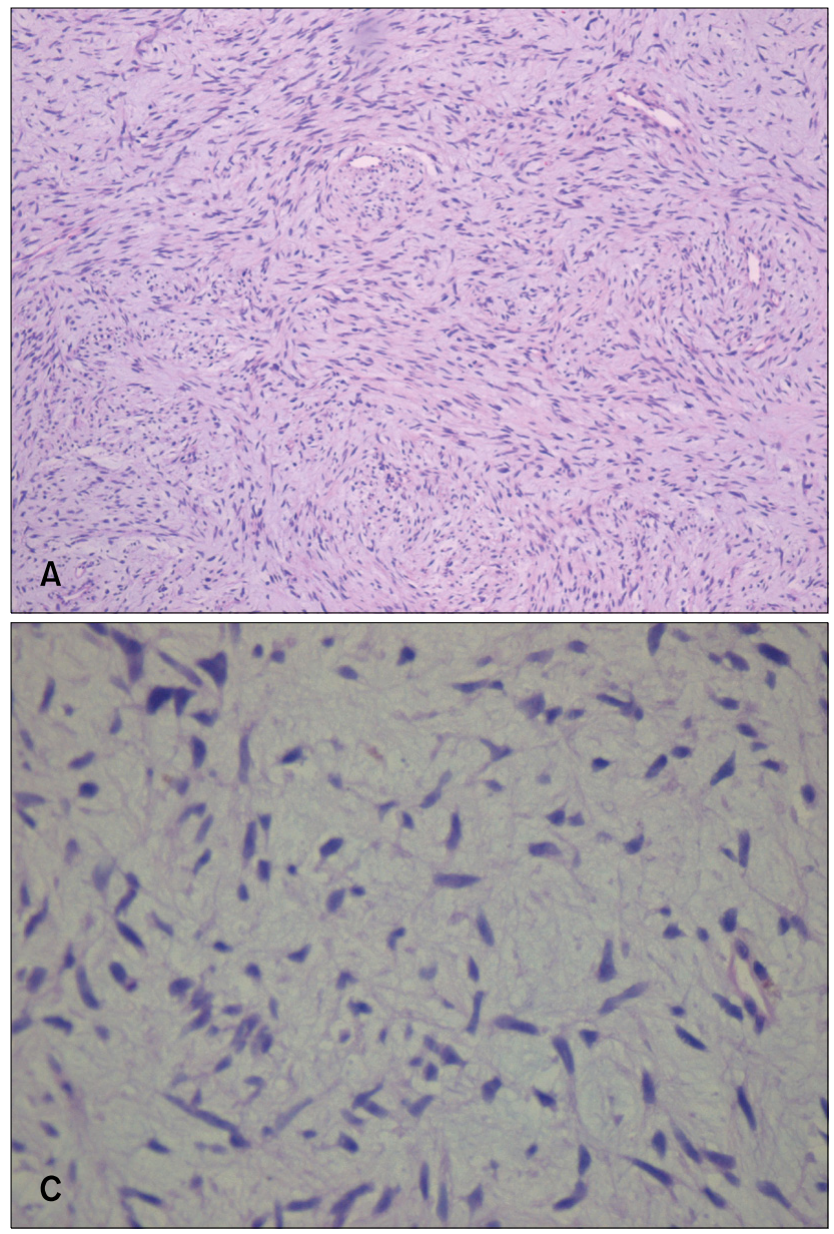

B

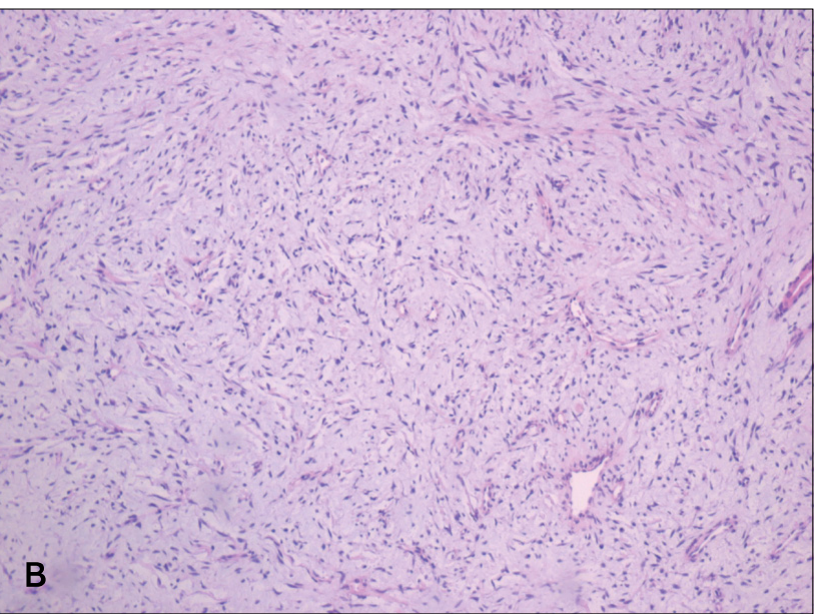

Fig. 3. (A) The tumor showed the characteristic storiform pattern in some parts of the lesion $(\mathrm{H} \& \mathrm{E}, \times 100)$. (B) In addition to the typical storiform pattern, prominent myxoid stromal changes were demonstrated $(H \& E, \times 100)$. (C) Bland spindle cells with oval nuclei were randomly embedded in the loose myxoid stroma $(\mathrm{H} \& \mathrm{E}, \times 400)$. 


\section{DISCUSSION}

DFSP is a slowly growing, but locally aggressive tumor, and it usually develops as a multinodular reddish-blue plaque on the trunk. Occurrence at sites of previous trauma has been reported, and many patients have a previous, long preoperative history ${ }^{1}$. Our patient had neither previous trauma nor operation history, except vaginal hysterectomy for cervical cancer, 16 years ago. Histologically, DFSP consists of relatively uniform spindle cells containing elongated nuclei, without significant cytologic atypia or pleomorphism, and arranged in a predominantly storiform typical pattern. Although the tumor is usually located in the dermis, it invariably shows the infiltrative growth pattern, with trapping of the subcutaneous fat tissue in the characteristic honeycomb appearance.

The myxoid DFSP is a rare variant of DFSP, characterized by the prominent myxoid stromal changes. Since the first case was cited in 1983 by Frierson and Cooper ${ }^{2}$, only a few cases have been reported in the literature. The pathogenesis of the myxoid change remains uncertain, and the majority of cases were presented with a slowly growing, firm subcutaneous mass. The most commonly involved sites were the extremities, followed by the head and neck ${ }^{3}$. In addition to the typical histological features of the ordinary DFSP, the tumor cells of the myxoid DFSP are embedded in an abundant, palely eosinophilic myxoid stroma, and prominent, thin-walled vessels are frequently present throughout the tumor ${ }^{3}$. Immunohistochimical findings are consistent with the typical DFSP, with the positive staining for CD34 ranging from $84 \%$ to $100 \%$, and negative for other markers, such as S-100, desmin and actin $^{4}$.

Prominent myxoid changes are often obscured by the typical storiform pattern, and this may cause considerable diagnostic problems, especially in the distinction between more and less aggressive myxoid mesenchymal neoplasms $^{5}$. The differential diagnosis of myxoid DFSP is diverse and includes myxoid liposarcoma, myxofibrosarcoma, myxoid neurofibroma and superficial angiomyxoma. Myxoid liposarcoma is mainly seen in deep soft tissues of the thigh and is distinguished from the presented case by the presence of lipoblasts and atypical, undifferentiated mesenchymal tumor cells, which are usually CD34 negative. Superficially located low-grade myxofibrosarcoma can be easily confused with the myxoid DFSP, because both consist of spindle-shaped cells embedded in a myxoid stroma, rich in small vessels. However, the lowgrade myxofibrosarcoma is a rather well-circumscribed tumor, with overall increased cellularity, compared with the myxoid DFSP, and the myxoid areas show a more whorled pattern. In addition, in many cases of low-grade myxofibrosarcoma ${ }^{6}$, the tumor cells are stained positively for the EMA. The myxoid neurofibroma can be distinguished from the presented case by the presence of S-100 protein positivity, wavy buckled nuclei and intralesional axons. The superficial angiomyxoma can be difficult to distinguish from the myxoid DFSP, because the former may also express CD34. However, in contrast to the myxoid DFSP, the superficial angiomyxoma lesions tend to be displayed in a lobular growth pattern, and scattered neutrophils surrounding the vessels are characteristically seen in a number of cases.

In summary, we presented an interesting case of myxoid DFSP, rarely reported in the Korean dermatology literature, and awareness of this rare variant of DFSP is important to avoid misdiagnosis of more or less aggressive myxoid tumors, which could lead to either under-treatment or overtreatment.

\section{REFERENCES}

1. McKee PH, Calonje JE, Granter S, Calonje E, Granter SR. Pathology of the skin: with clinical correlations. Vol 2. Philadelphia, Pa: Elsevier Mosby, 2005.

2. Graadt van Roggen JF, Hogendoorn PC, Fletcher CD. Myxoid tumours of soft tissue. Histopathology 1999;35: 291-312.

3. Reimann JD, Fletcher CD. Myxoid dermatofibrosarcoma protuberans: a rare variant analyzed in a series of 23 cases. Am J Surg Pathol 2007;31:1371-1377.

4. Haycox CL, Odland PB, Olbricht SM, Piepkorn M. Immunohistochemical characterization of dermatofibrosarcoma protuberans with practical applications for diagnosis and treatment. J Am Acad Dermatol 1997;37:438-444.

5. Mentzel T, Schärer L, Kazakov DV, Michal M. Myxoid dermatofibrosarcoma protuberans: clinicopathologic, immunohistochemical, and molecular analysis of eight cases. Am J Dermatopathol 2007;29:443-448.

6. Mertens F, Fletcher CD, Antonescu CR, Coindre JM, Colecchia M, Domanski HA, et al. Clinicopathologic and molecular genetic characterization of low-grade fibromyxoid sarcoma, and cloning of a novel FUS/CREB3L1 fusion gene. Lab Invest 2005;85:408-415. 